\title{
„Klima nervt!“ - Zum didaktischen Umgang mit Widerständen bei der Thematisierung des Klimawandels im Religionsunterricht
}

\author{
Annika Krahn ${ }^{a}$ Alexander Schimmel ${ }^{b}$ \\ aUniversität zu Köln; bJohannes Gutenberg-Universität Mainz
}

Kontakt: aschimme@uni-mainz.de

eingereicht: 05.06.2021; überarbeitet: 15.08.2021; angenommen: 27.08.2021

\begin{abstract}
Zusammenfassung: Ökologische Problemstellungen, insbesondere der Klimakrise, nehmen zunehmend größeren Raum in öffentlichen Debatten und Diskursen ein. Dabei zeigen sich divergierende gesellschaftliche Positionen in der Bewertung der Relevanz und der zu ergreifenden Gegenmaßnahmen. Wie in der Gesellschaft, so zeigen auch Schüler*innen im Religionsunterricht ein heterogenes Tableau an Einstellungen. Der Beitrag beleuchtet Einstellungen, die einer hohen (Lern-)Motivation entgegenstehen und offeriert (religions-)didaktische Umgangsweisen.

Schlagwörter: Einstellungen, Klimaskepsis, Jugend-Studie, Religionsunterricht, religiöse Bildung für nachhaltige Entwicklung

Abstract: Ecological problems, especially the climate crisis, play a prominent role in public discussion and discourses. Several social positions appear within the evaluation of the pertinence and distinct counteractive measures. As in public, students of religious education show a variety of attitudes. This paper presents attitudes, which avoid high learning motivation and offers didactic answers.

Keywords: attitude, climate-scepticism, youth studies, religious education, religious education for sustainable development
\end{abstract}

\section{Einführung}

Rekordzahlen bei der Klimademo im September 2019 - Deutschlandweit haben 1,4 Millionen Menschen bei Fridays for Future mitgemacht, mehrheitlich Schüler*innen. Viele von ihnen sind begeistert, wenn nun der Klimawandel auch im Religionsunterricht als religiöse Bildung für nachhaltige Entwicklung thematisiert wird; andere sind skeptisch und fragen, ob das (ggf. schon wieder) sein muss oder haben Zweifel an der Relevanz des Themas bzw. an der Sinnhaftigkeit der Behandlung des Themas im schulischen Unterricht, insbesondere im Religionsunterricht. Zugegebenermaßen müssen einige Faktoren stimmen, damit das Thema auf offene Schüler*innenohren stößt: Der Klimawandel muss als reale Bedrohung betrachtet werden und der didaktische Aufgriff als sinnvoll gesehen werden, etwa um sachlich dabei etwas zu lernen, aber auch um aus der Beschäftigung Folgerungen zur Abhilfe ziehen zu können. Neben der Aufgeschlossenheit der Schüler*innen gegenüber dem Thema bedarf es auch der Einschätzung, dass der Religionsunterricht einen geeigneter Raum für die Thematisierung bietet. Bei Fehlen dieser (oder einiger dieser) Einschätzungen ist bei Schüler*innen mit Widerständen zu rechnen.

Vor dem Hintergrund etwa der Unwetterkatastrophe im Juli 2021 mit Überschwemmungen und vielen Toten in Rheinland-Pfalz und Nordrhein-Westfalen, deren Intensität und Wahrscheinlichkeit in engem Zusammenhang mit der Klimakrise stehen, wird erneut die bildungspolitische und religionsdidaktische Notwendigkeit der Thematisierung des Klimawandels deutlich. „Wenn religiöse Bildung konsequent subjektorientiert sein will, muss sie den Klimawandel thematisieren, denn er wird das Leben aller Schülerinnen und Schüler bestimmen. Ökologische Verantwortung ist die zentrale ethische und politi- 
sche Frage unserer Zeit und gehört als solche auch in den Religionsunterricht" (Bederna \& Krahn, 2021, S. 8; auch Bederna, 2020b).

Der vorliegende Beitrag nimmt verschiedene Beweggründe für eine von Widerständen geprägte Begegnung in den Blick, sucht nach soziologisch-empirischen Hinweisen hierauf, fasst die auf Schüler*innenseite vorliegenden Haltungen mit dem sozialpsychologischen Begriff der Einstellung und sucht nach psychologisch reflektierten Möglichkeiten des didaktischen Umgangs.

\section{Jugendsoziologischer Blick auf Einstellungen gegenüber dem Klimawandel und dem Reli- gionsunterricht}

Eine von der Organisation Wissenschaft im Dialog (WiD) erhobene Umfrage verdeutlicht, dass knapp $30 \%$ der deutschen Bevölkerung wissenschaftlichen Fakten zum anthropogenen Klimawandel mindestens teilweise misstraut (WiD, 2016, S. 13; Brunnengräber, 2013). Dass ebenfalls eine nennenswerte Minderheit der Jugendlichen den anthropogenen Klimawandel zumindest in Zweifel zieht, illustrieren Studien über Einstellungen von Kindern und Jugendlichen in Bezug auf Bedrohungslage und Verantwortung des Klimawandels: Sowohl die jüngste Shell-Studie aus dem Jahr 2019, die Studie „Zukunft? Jugend fragen!“ im Auftrag des Bundesumweltministeriums von 2019 und 2020 sowie die im gleichen Jahr erfolgte Sinus-Studie zu „Fridays for Future und Klimaschutz“ zeigen, dass rund 2/3 der Jugendlichen den Klimawandel als besonders bedrohlich oder eher bedrohlich wahrnehmen - wobei sich weibliche Jugendliche mit $74 \%$ noch etwas stärker sorgen als männliche Jugendliche mit $62 \%$. Ca. $20 \%$ der Jugendlichen gehören zu sog. Klimaleugner*innen: Sie glauben nicht, dass Menschen schuld am Klimawandel seien (Sinus, 2019, S. 12). Diese Zahl korrespondiert mit dem Ergebnis der aktuellen Studie des Bundesministeriums für Umwelt und Naturschutz, denn ca. 20 \% der Jugendlichen geben hier an, dass Umwelt- und Klimaschutz überhaupt nicht wichtig seien (BMU, 2020, S. 16).

Von der großen Mehrheit der Jugendlichen, die den Klimawandel als (besonders) bedrohlich wahrnehmen, sind sich 2/3 darin einig, dass insbesondere die Politik in die Pflicht zu nehmen sei, um den Klimawandel zu bekämpfen und "nur" 49\% der Jugendlichen sehen die Wirtschaft bei der Eindämmung des Klimawandels in der Verantwortung (BMU, 2020, S. 16). Sich selbst sieht die junge Generation eher selten in der Pflicht (26\%) (Sinus, 2019, S. 10). Diese Haltung bestätigt auch eine Studie des Wuppertal Instituts in Zusammenarbeit mit Ebay-Kleinanzeigen: Nur 49\% der 19- bis 29-jährigen stimmen der Aussage zu: „Ich achte im Alltag sehr darauf, Ressourcen zu schonen.“ Jede fünfte Person möchte keinerlei Abstriche für Umwelt und Klima beim privaten Konsum machen (Wilts \& Fecke, 2020, S. 25). Dies ist vergleichbar mit den Ergebnissen der aktuellen Studie des Bundesumweltministeriums. Ca. 12\% der befragten Jugendlichen geben hier an, deshalb nicht bei der Protestbewegung „Fridays for Future“ mitzumachen, da sie sowieso nichts bewegen könnten (BMU, 2020, S. 25). Dass die Handlungsmotivation deutscher Jugendlicher im Vergleich mit den europäischen Nachbarn am geringsten ausgeprägt ist, ergab eine Zusatzbefragung der PISA-Studie 2018 in der Domäne "Global Competence“ (2019). Derzufolge können deutsche Jugendliche globale Probleme wie beispielsweise die Klimakrise zwar besonders gut erklären, sie glauben jedoch am wenigsten, dass sie die globalen Probleme auch selbst ändern können, und so boykottieren nur ca. 19\% der deutschen Jugendlichen „Produkte oder Firmen aus politischen, ethischen oder ökologischen Gründen“ (Weis et al., 2020, S. 21).

Zusammenfassend ergibt sich folgendes Bild: Während ca. 2/3 der Jugendlichen in Deutschland zwar bestätigen, dass der Klimawandel eine Krise mit globalem Ausmaß ist, die durchaus sehr beängstigend ist, sind nur ca. 1/5 davon bereit, klimaschützende Maßnahmen zu ergreifen, da allein Politik und/oder Wirtschaft in die Pflicht genommen werden, die Klimakrise abzuwenden. Ebenfalls 1/5 der Jugendlichen sehen auch sich selbst in der Verantwortung und verändern nach eigener Auskunft ihr eigenes Handeln entsprechend (BMU, 2018, S. 61). 1/5 der Jugendlichen können die zukunftsgefährdenden Auswirkungen des Klimawandels überhaupt nicht bestätigen. 
Im Rahmen der vorliegenden Untersuchung werden neben diesen soziologischen Hinweisen zu Umweltfragen auch Einstellungen gegenüber dem Religionsunterricht in den Blick genommen, um ein vollständiges Gesamtbild möglicher Lernhindernisse entstehen zu lassen.

Bereits die ersten empirischen Studien zum Religionsunterricht aus Schüler*innenperspektive aus den 1970er-Jahren bestätigen, dass nicht einmal 10\% der Jugendlichen ihren Religionsunterricht als „sehr wichtig" empfinden (Prawdzik, 1973, S. 149; Bucher, 2019, S. 38) und die große Mehrheit ihre Zeit mit dem RU verschwendet sehen (Havers, 1972). Diese frühen Zahlen bestätigt eine Studie aus dem Jahr 2000, die zudem mit steigendem Alter ein abnehmendes Interesse an dem Religionsunterricht erkennbar macht (Bucher, 2001, S. 50f.). Dies und die darin enthaltene Infragestellung des Religionsunterrichts im schulischen Kontext illustriert auch eine 2019 herausgegebene Studie des Comenius-Instituts (Comenius, 2019, S. 151). Auch wenn die KMK in Bezug auf den evangelischen Religionsunterricht lediglich eine stabile Abmeldequote von bundesweit unter 5\% dokumentiert (KMK, 2002, S. 18), rangiert der konfessionelle Religionsunterricht mit Blick auf die Beliebtheit der schulischen Unterrichtsfächer im hinteren Drittel (Bucher, 2001, S. 50f.). Zwar seien Ritzer zufolge ca. 60\% der Schüler*innen "grundsätzlich am RU interessiert" (Ritzer, 2010, S. 193; auch in Bucher, 2019, S. 40), aber es bestehe eben kein besonderes Interesse am Religionsunterricht. Diese Ergebnisse korrespondieren mit der Bestrebung der rheinland-pfälzischen Landesschüler*innenvertretung aus dem Jahr 2019, den konfessionellen Religionsunterricht abzuschaffen.

Monika Fuchs kann in diesem Zusammenhang u. a. vor dem Hintergrund der YouGov-Studie aus dem Jahr 2016 und der „Auswertung RU 2015/2016“ der KMK 2016 Folgendes feststellen: „Es ist mit einem rückläufigen Belegungsinteresse am evangelischen bzw. am katholischen Religionsunterricht zu rechnen. Gegenläufig hierzu dürften sich Engagement und Leidenschaft aufseiten der Kritiker und Gegner des Religionsunterrichts eher noch steigern, die Anfragen an Art. 7 GG zunehmen. Mit einer Verschärfung der gesellschaftlichen und politischen Debattenlage ist zu rechnen“ (Fuchs, 2019, S. 99).

Vor dem Hintergrund erodierender Akzeptanz eines konfessionellen Religionsunterrichts und dem schwindenden Verständnis für seinen (religionsdidaktisch wohlbegründeten) Sinn ist damit zu rechnen, dass zu den beiden oben genannten Einstellungselementen eine Einstellung die offene Thematisierung der Klimakrise im Unterricht erschwert: Nicht wenige Schüler*innen erkennen zwar die Klimakrise als epochales Problem an und betrachten auch ihr eigenes Handeln als relevant, haben jedoch aufgrund ihrer Einstellung gegenüber dem Religionsunterricht wenig Verständnis dafür, dass das Thema ausgerechnet in diesem Fach besprochen werden soll. Eine stichprobenartige Umfrage an einem Kölner Gymnasium im Frühjahr 2021 in der 8. Jahrgangsstufe und in der Oberstufe hat diesen Eindruck bestätigt.

\section{Themenbezogene Einstellungen von Schüler*innen als didaktische Kategorie}

Mit dem hier gelegten Fokus auf Einstellungen, die die offene und lernbereite Thematisierung des Klimawandels erschweren, lassen sich aus den soziologischen Befunden drei Einstellungen bzw. Einstellungselemente identifizieren, die alle drei unabhängig voneinander vertreten werden können. Schüler*innen können alle, einzelne oder (mehrheitlich vielleicht sogar) keine davon tragen; diese stellen mögliche Elemente eines holistischen Einstellungsnetzwerks (s. u.) dar - keinesfalls wollen sie als Typologie von Schüler*innen verstanden sein.

1. Klimakrise und Klimaschutz werden im Religionsunterricht als irrelevante Themen erachtet, weil die Schüler*innen bezweifeln oder skeptisch sind, dass der Klimawandel überhaupt wirklich besteht, dass er anthropogene Ursachen hat oder dass er als relevantes ökologisches und soziales Problem zu betrachten ist.

2. Die Klimakrise wird mit all ihren verheerenden Folgen anerkannt oder nicht, jedenfalls wird die per- 
sönliche Verantwortlichkeit i. S. der Zuständigkeit oder auch mangels Selbstwirksamkeit die Möglichkeit negiert, durch eigenes Verhalten Einfluss auf die Krise zu nehmen - Mitigation ist die Aufgabe anderer.

3. Unabhängig vom Vorliegen von Problembewusstsein und Selbstwirksamkeit - der Religionsunterricht jedenfalls wird als ungeeignet empfunden, dieses Thema zum Unterrichtsgegenstand zu erheben.

Mit dem Verständnis dieser Positionen als Einstellungen werden durch Erfahrung vorstrukturierte, relativ dauerhafte Reaktionsdispositionen gegenüber bestimmten Gegenständen im weitesten Sinn gemeint. Im Zentrum steht die Bewertung eines Einstellungsobjekts, das Personen, Sachverhalte, Objekte oder Ideen sein können, so also auch gegenüber dem Gegenstand Klimawandel, gegenüber RU oder gegenüber der Idee, Klimawandel im RU zu behandeln (Werth, Denzler \& Mayer, 2020, S. 243). Neben diesem affektiven Bewertungsaspekt umfassen Einstellungen stets auch Handlungstendenzen (im Wesentlichen die zur Zu- bzw. Abwendung) wie auch alles Kognitive, gesammelte Informationen, Wissen und objektbezogene Meinungen. Einstellungen stehen selten isoliert, sondern bilden insbesondere durch häufige ,Aktivierung' sog. Knoten in assoziativen Netzwerken (Werth et al., 2020, S. 25). Die kognitiven Elemente bilden einen ,psycho-logischen' Zusammenhang. Stehen sie im inhaltlichen Widerspruch, tritt kognitive Dissonanz ein, was als unangenehm erlebt wird. Die Wiederherstellung kognitiver Konsistenz ist häufig die Ursache von Einstellungsänderungen (Werth et al., 2020, S. 261). Hierbei gelten auch eigene Handlungen (insbesondere solche, die mit Regelmäßigkeit vorgenommen werden) als Element, das nicht dauerhaft in Spannung zu anderen Einstellungselementen stehen soll. Bei Einstellungen zum Klimawandel sind wohl vielen dissonante Wahrnehmungen wie diese vertraut: Wir wissen um die Schädlichkeit von Verbrennungsmotoren und nutzen doch private Pkw. Der sog. Mind-BehaviorGap, also die Kluft zwischen Wissen bzw. Problembewusstsein und (Nicht-)Verhalten, ist durch die Umweltpsychologie gut erforscht (Enzian, 2015). Zur ,Glättung' nutzen wir gerne entlastende - zum tatsächlichen Verhalten passende und dieses rechtfertigende - kognitive Elemente und suchen durchaus gezielt nach diesen.

Einstellungen übernehmen die wichtige kognitive Funktion, gegenüber Sachverhalten automatisch bereits gefasste Urteile abzurufen, ohne Details merken, prüfen bzw. erneut abrufen zu müssen - sie erlauben den aufwandsarmen Rekurs auf bewährte Reaktionsregeln. „Klimawandel? Alles Quatsch!“ ist deshalb - solange sich dieses Urteil konsistent ins Gesamtnetz einfügt - eine ,nützliche' Einstellung, die dazu dient, lästige Anfragen auf Abstand zu halten. Zugleich übernehmen Einstellungen motivationale Funktionen, da sie unser Selbstwertgefühl steigern können, wenn wir uns in Einklang mit ihnen verhalten, bzw. einen sozialen Identitätsgewinn ermöglichen, wenn wir uns über Einstellungen mit anderen Personen oder Gruppen identifizieren.

Schüler*innen als Träger von Einstellungen zu sehen, verändert den Blick auf die Ziele und die zu erwartende unterrichtliche Interaktion: Einerseits wächst durch das Wissen um die Stabilität und die weitgehend unbewusst laufenden Mechanismen verzerrender Informationsverarbeitung zur Aufrechterhaltung das Problembewusstsein für den zu antizipierenden Effekt auf die Änderungen von Einstellungen; andererseits zeigen sich auch gezielte Möglichkeiten, die Änderungen von Einstellungen durch Bildungsprozesse anzuregen.

Zunächst drängt sich jedoch die Frage nach der bildungstheoretischen Legitimität pädagogischer Persuasionsversuche: Soll Unterricht versuchen, Schülereinstellungen zu ändern, und wenn ja, mit welchen Mitteln?

Sowohl aus (religions-)pädagogischen (Überwältigungsverbot, Kontroversitätsgebot) wie auch (schul-) psychologischen Gründen (Vermeidung von Reaktanz, also dem inneren Widerstand gegen den subjek- 
tiv-erlebten Versuch der Einschränkung von Handlungs- oder Meinungsfreiheit) ist auf manipulative Strategien zu verzichten und auf transparente Kommunikation zu achten, die sich auch in der Differenzierung von Sachinformation und erkennbarer Position (etwa der Kirche oder der Lehrperson) zeigt. Meinungs- und Urteilsfreiheit gilt es zu wahren und ggf. zu verteidigen. Dennoch gilt es bildungstheoretisch fundierte Ziele und Kriterien wünschenswerter Merkmale von Einstellungen zu entwickeln und ggf. im Unterricht transparent zu machen.

Als unbestrittenes Bildungsziel kann hierfür bei der Selbstbestimmung angesetzt werden. Diese setzt im Anschluss an Heinrich Roth Selbst-, Sach- und Sozialkompetenz voraus, weshalb Bildung die Förderung dieser Bereiche zu betreiben hat (Roth, 1971, S. 180). Wissensaneignung (z. B. über den Klimawandel), Meinungs- und Urteilsbildung wie auch die Ausbildung von Werten zu fördern und anzuregen, sind anerkannte Bestandteile verbreiteter Zieltaxonomien. Gefasst in der Kategorie von Einstellungen lassen sich diese reformulieren: Unterricht zielt auf die Ausbildung von Einstellungen mit drei Merkmalen: Sie sollen (a) den Schüler*innen klar und bewusst (explizit) sein, sie sollen (b) den Einstellungsgegenständen gegenüber sachgerecht und differenziert sein und die Schüler*innen sollen (c) eine kritische Haltung gegenüber den eigenen Einstellungen einnehmen, d. h. sie sollen bereit sein, in Auseinandersetzung mit Anfragen diese in Frage stellen zu lassen, gerade angesichts kursierender Klimaleugnungspositionen stellt dieser Punkt eine besondere Herausforderung an das Ganze auf Wissenschaft und Rationalität gründendem Bildungswesen dar. Zu jedem Merkmal passen bestimmte unterrichtliche Aktivitäten, die zur Förderung beitragen, wobei diese idealtypisch unterschieden und insbesondere in der Praxis selten klar voneinander abzugrenzen sind:

\begin{tabular}{|l|l|l|}
\hline & Angezielte Einstellungsmerkmale: & Entwicklungsförderliche Unterrichtshandlungen: \\
\hline a & bewusst, explizit & klären \\
\hline b & sachgerecht, angemessen, differenziert & informieren, präzisieren, Vorstellungen weiten \\
\hline c & $\begin{array}{l}\text { selbstkritisch (kritisch gegenüber den } \\
\text { eigenen Einstellungen), diskursbereit }\end{array}$ & $\begin{array}{l}\text { konfrontieren, herausfordern, irritieren, sensibilisieren, zu } \\
\text { neuen Sichtweisen einladen, bewusstmachen von Prozes- } \\
\text { sen der Meinungsbildung (insb. über soziale Medien) }\end{array}$ \\
\hline
\end{tabular}

Tab. 1: Einstellungsmerkmale und entwicklungsförderliche Unterrichtshandlungen, (Schimmel, 2009, S. 243)

\section{Ideen für den gezielten religionsdidaktischen Umgang}

Wenden wir die drei förderungswürdigen Einstellungsmerkmale $(a, b, c)$ auf die drei o. g. möglichen Schüler*inneneinstellungen $(1,2,3)$ an, die eine unvoreingenommene Auseinandersetzung mit dem Thema Klimawandel im Religionsunterricht erschweren, ergibt sich folgende Matrix, die jedem Entwicklungsfeld geeignete Aufgaben und Inhalte zuordnet, wobei insbesondere die Grenze zwischen den Inhalten der Spalten b und c fließend sind (siehe Seite 91).

(ad 1) Was bedeutet die erstgenannte Schüler*inneneinstellung für die konkrete religionsdidaktische Umsetzung? Ein mangelndes Problembewusstsein in Bezug auf die Klimakrise zeichnet die erste Gruppe der Einstellungen auf Schüler*innenseite aus. Dies kann dem bloßen Desinteresse oder aber der Skepsis gegenüber der Erkenntnis des menschengemachten Klimawandels geschuldet sein; hierbei stehen nicht selten auch spezifische Formen von Wissenschaftsleugnung im Hintergrund. Diese zeichnet sich dadurch aus, dass sie fundierte wissenschaftliche Aussagen negiert, auch wenn viele Theorien der Wissenschaft in Bezug auf den anthropogenen Klimawandel sehr gut belegt sind, wie die Tatsache, dass die Menschheit seit der industriellen Revolution erheblich dazu beitrug, dass die Konzentration von Treibhausgasen in der Atmosphäre und damit auch die globale Temperatur stetig ansteigt (Bederna \& 
Krahn, 2021). Dies und die dramatischen Folgen dessen werden erneut in dem jüngsten Sachstandsbericht des IPCC deutlich (Masson-Delmotte et al., 2021).

Das Streuen von Fehlinformationen, Diskreditierung von Forschenden oder die Akzeptanz von Verschwörungstheorien können Folgen einer solchen Wissenschaftsleugnung sein (Rumberger, 2021, S. 7f.). Konsistent durchdacht sind solche Positionen i. d. R. nicht, infolgedessen lohnt es, die subjektiven Theorien der Schüler*innen (als zu wissenschaftlichen Theorien korrespondierende) herauszuarbeiten, indem die Einstellungen versprachlicht, sachlich freigelegt und geklärt werden (a).

Die Vermittlung gesicherter naturwissenschaftlicher Erkenntnisse zum Klimawandel (etwa in den naturwissenschaftlichen Fächern) ist ein notwendiges kognitives Element zu einer problembewussten Einstellung gegenüber dem Klimawandel (b). Ferner ist ein Blick auf die aktuelle Entwicklung in der Wissenschaftskommunikation unabdingbar. Ging man vor dem PISA-Schock mehr oder weniger davon aus, dass lediglich die richtigen Informationen zu einem besseren Verständnis einer Thematik führen, so ist vor dem Hintergrund psychologischer Mechanismen und sozialpsychologischer Aspekte ein besseres Verständnis der individuellen Bildungsprozesse erkennbar geworden. Menschen vertreten bestimmte Thesen oder Standpunkte im Rahmen der Anerkennung in der Peer-Group, auch wenn diese dem wissenschaftlichen Konsens widersprechen (Schmid, 2020). Dies ist vergleichbar mit der Theorie der gruppenabhängigen Risikowahrnehmung. Ihr zufolge ist die eigene Risikowahrnehmung weniger abhängig von objektiv-wissenschaftlichen Erkenntnissen, sondern von der gruppenabhängigen Risikowahrnehmung (vgl. die aktuelle Studie zur Risikowahrnehmung einer COVID-19 Erkrankung in: Gerhold, 2020). Dies bedeutet also nicht, dass wissenschaftlich fundierte Ergebnisse aus dem Grund für beachtenswert gehalten werden, weil die Validität und die Reliabilität dieser hinreichend geprüft worden sind, sondern weil der Standpunkt der Gruppe die Akzeptanz der Erkenntnisse befördert.

Für den Religionsunterricht heißt das, dass die Schüler*innen mit den sozialpsychologischen Prozessen der Meinungsbildung (z. B. der Funktionsweise von Filterblasen) bekannt gemacht werden sollen (c). Auch für die Unterrichtenden ist es hilfreich, von der Genese der Einstellungen ihrer Schüler*innen zu erfahren, um Brücken bauen zu können. So lässt sich beispielsweise mit eher konservativen Schüler*innen ein Gespräch über die Klimakrise führen, ohne ihr konservatives Wertesystem ins Wanken zu bringen, indem Sicherheit, Risikoaversionen, Pragmatismus oder der Wunsch zur Schöpfungsbewahrung zum Tragen kommen. In diesem Zusammenhang und als weiterer Ideengeber bietet sich die COIN-Studie 2014 an, die die Wichtigkeit von Werten und das unterschiedliche Framing der Klimakrise junger Menschen gewinnbringend untersucht hat. Nach einer Phase der Selbstklärung in Einzelarbeit und Kleingruppen ist deshalb ein offenes Eingehen auf die von der Lerngruppe geäußerten Aspekten wichtig, um dann auch gezielt Diskursimpulse bieten zu können. Sollen widersprüchliche Haltungen populistischer Meinungsmacher bzgl. des Klimawandels aufgedeckt und entlarvt werden, ist es auch wichtig, die Emotionen, die gezielt angesprochen werden, ernst zu nehmen und diese „durch apokalyptische Untergangsszenarien nicht noch zu befeuern" (Kommission Weltkirche der Deutschen Bischofskonferenz, 2021, S. 60). Diese Vorstellungen apokalyptisch-endzeitlichen Ausmaßes sind allerdings seitens der Klimatologie nicht von der Hand zu weisen (Masson-Delmotte et al., 2021), sodass von Klimaskeptikern*innen auch häufig die Reformation bzw. die Abschaffung der "Klima-Religion“ gefordert wird. Nun enthält die Rede über den Klimaschutz durchaus wert- und sinnreflexive und somit auch religiöse Elemente. Das Problem liegt aber darin, „dass die Skeptiker(innen) Religion ohne jede nähere Reflexion und Begründung (normativ gesehen) negativ werten“ (Rosenberger, 2014, S. 96). Demzufolge sollte vor allem in der Auseinandersetzung mit Klimaskeptiker*innen vor dem Hintergrund der oben genannten COIN-Studie 2014 der Wertehorizont einbezogen und thematisiert werden (Rosenberger, 2014, S. 96). 


\begin{tabular}{|c|c|c|c|}
\hline & \multicolumn{3}{|c|}{ Angezielte Einstellungsmerkmale und mögliche didaktische Maßnahmen } \\
\hline $\begin{array}{l}\text { Schüler*innen- } \\
\text { Einstellung }\end{array}$ & $\begin{array}{l}\text { a. bewusst, } \\
\text { geklärt }\end{array}$ & $\begin{array}{l}\text { b. sachgerecht, } \\
\text { differenziert }\end{array}$ & $\begin{array}{l}\text { c. selbstkritisch, } \\
\text { diskursbereit }\end{array}$ \\
\hline $\begin{array}{l}\text { 1. mangelndes Pro- } \\
\text { blembewusstsein } \\
\text { (gegenüber dem } \\
\text { Klimawandel) }\end{array}$ & \multirow{3}{*}{ 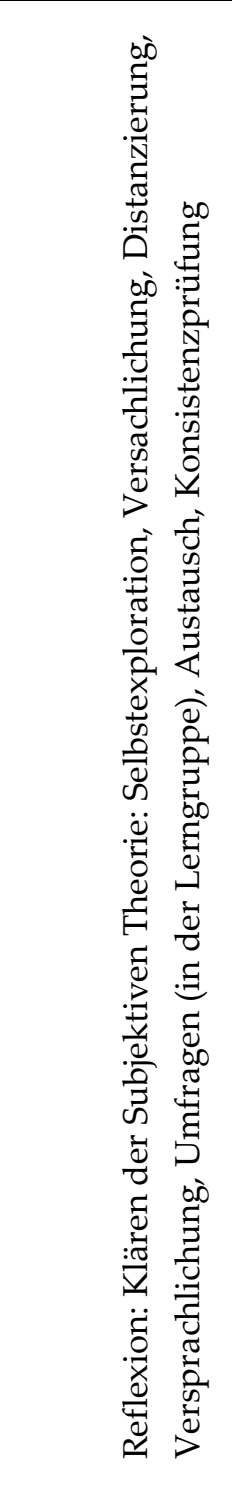 } & $\begin{array}{l}\text { Klärung und Aus- } \\
\text { tausch über gesicherte } \\
\text { naturwissenschaftliche } \\
\text { Erkenntnisse zum Kli- } \\
\text { mawandel; Kriterien } \\
\text { rationaler Geltungs- } \\
\text { ansprüche }\end{array}$ & $\begin{array}{l}\text { Problematisierung } \\
\text { von medialen und } \\
\text { sozial-psychologi- } \\
\text { schen Effekten (z. B. } \\
\text { Filterblasen...) sowie } \\
\text { von antirealistischen } \\
\text { Strategien der Klima- } \\
\text { skeptiker }\end{array}$ \\
\hline $\begin{array}{l}\text { 2. Zweifel an der ei- } \\
\text { genen Verant- } \\
\text { wortlichkeit, } \\
\text { mangelnde Selbst- } \\
\text { wirksamkeit }\end{array}$ & & $\begin{array}{l}\text { Analyse von indivi- } \\
\text { dual- und sozial-ethi- } \\
\text { scher Ebene; Klimage- } \\
\text { rechtigkeit; quantitati- } \\
\text { ves Bewusstsein (z. B. } \\
\text { ökolog. Fußabdruck) }\end{array}$ & $\begin{array}{l}\text { Konfrontation/Begeg- } \\
\text { nung an globalen } \\
\text {,Rändern', Empathie / } \\
\text { Reflexion von Nor- } \\
\text { malitätsvorstellung; } \\
\text { Vorbilder/ Begeg- } \\
\text { nungslernen/ Bewe- } \\
\text { gungen (IST-Normen); } \\
\text { Identität/ Selbstwirk- } \\
\text { samkeitserleben }\end{array}$ \\
\hline $\begin{array}{l}\text { 3. Skepsis bzgl. Zu- } \\
\text { ständigkeit und } \\
\text { Kompetenz von } \\
\text { RU (inkl. Sorge } \\
\text { vor } \\
\text { Moralisierung) }\end{array}$ & & $\begin{array}{l}\text { Aufweis thematischer } \\
\text { Bezug Theologie und } \\
\text { Nachhaltigkeit: Ver- } \\
\text { hältnis Mensch-Natur } \\
\text { (Anthropozän), u. So- } \\
\text { zialethik (Reich-Gottes- } \\
\text { Botschaft, strukturelle } \\
\text { Sünde) }\end{array}$ & $\begin{array}{l}\text { theol. Anthropologie } \\
\text { (Deskription und Vi- } \\
\text { sion) u. Spiritualität: } \\
\text { Umgang mit eigenen } \\
\text { Grenzen/Scheitern } \\
\text { und schuldhafter Ver- } \\
\text { strickung, Emotionen; } \\
\text { „Schöpfungslust statt } \\
\text { Ökofrust“; Theologi- } \\
\text { zität des Subjekts / } \\
\text { Bonhoeffer: Verant- } \\
\text { wortungsethik) }\end{array}$ \\
\hline
\end{tabular}

Tab. 2: Einstellungen, Zieldimensionen und didaktische Maßnahmen

(ad 2) Ein Hemmnis der offenen (auch religionsunterrichtlichen) Auseinandersetzung mit ökologischethischen Themen stellt insbesondere beim Klimawandel der Zweifel an der eigenen Verantwortung dar. Eine didaktische Begegnung auf diese zu Skepsis oder gar Fatalismus führenden Konzept der mangelnden Selbstwirksamkeit beginnt mit (a) methodischen Hilfen zur Klärung der eigenen Position, auf die eine inhaltliche Auseinandersetzung folgen kann. Hier geht es zunächst (b) um die sachliche Auseinandersetzung mit den aufgeworfenen Anfragen. Grundlegend ist die Erkenntnis, dass der Prozess der Transformation ursprünglich in dem Menschen selbst angelegt ist. Als Spiegel der menschlichen Vergänglichkeit ist die Transformation somit genuiner Teil der göttlichen Schöpfungsordnung. Dies in Rechnung gestellt, fällt die Aufgabe der Differenzierung von Individual- und Sozialethik direkt ins Fachcurriculum, die sich anhand des Klimaengagements didaktisch günstig veranschaulichen lässt. Das komplexe Verhältnis der beiden voneinander abhängenden Ebenen gilt es zu reflektieren, was zu Tage fördern sollte, dass die beiden Felder des Engagements komplementär zueinanderstehen und eine Al- 
ternative, etwa die der Diskreditierung von politischem Engagement in der FFF-Bewegung mit Verweis auf (tatsächliches oder vermeintliches) mangelndes persönliches Engagement der Aktivisten die Komplexität der Problematik verkennt. Als theoretischer Leitgedanke einer ethischen Bewertung kann das Konzept der $\mathrm{CO}_{2}$-Gerechtigkeit vorgestellt und diskutiert werden, nachdem weltweit egalitär ein ProKopf-Emissionsrecht vertreten wird (Vogt, 2021, S. 386).

Um das Thema Verhaltensänderung (hin zu mehr Nachhaltigkeit), das als abstraktes Ganzes eventuell erschlagend und abwegig scheint, zugänglicher und konkreter zu machen, sind Modelle der Quantisierung hilfreich, um zu verdeutlichen, dass auch kleine Schritte, z. B. die Reduktion des persönlichen ökologischen Fußabdrucks von (den für Deutschland durchschnittlichen Wert von) 4,8 gha auf 4,3 gha ein (motivierender) Anfang (!) sind, denn jede Maßnahme der Mitigation zählt. Zudem verdeutlichen die konkreten Fragen eines solchen Tests (wie er z. B. von der Organisation „Brot für die Welt“ angeboten wird) die relevanten Bereiche (Ernährung, Wohnen, Konsum, Mobilität im Alltag und bei Urlaubsreisen u. a.), aus denen man sich gezielt einzelne Bereiche für ein zeitlich begrenztes persönliches Experiment veränderten Verhaltens auswählen kann. Schwarz-weiß-Urteile lassen sich so auflösen und konkrete Vorhaben in realisierbare Reichweite rücken. Eine sinnvolle Ergänzung zur Ermittlung des ökologischen Fußabdrucks ist zudem die des ökologischen Handabdrucks, der Möglichkeiten positiver, insb. zivilgesellschaftlich-politische Aktivitäten auf dem Weg zu einer nachhaltigeren Gesellschaft, in den Blick nimmt (Reif \& Heitfeld, 2015).

Als dritten Schritt (c) auf der Grundlage einer differenziert-sachlichen Einschätzung ist nach passenden herausfordernde Anfragen oder gar Erfahrungen zu suchen, die geeignet sind, Einstellungen auf mehr Hinterfragbarkeit hin zu ,lockern': Zu denken ist beispielsweise an Begegnungserfahrungen: Wenn die aus vielerlei Gründen nicht einfache Herausforderung gelingt, authentische Begegnung mit Gleichaltrigen aus dem Globalen Süden zu ermöglichen, die von ihrer Lebenssituation berichten, kann dies helfen, die eigene Sicht auf das, was ,normal' ist, in Frage zu stellen: Was brauche ich wirklich? Welche (z. B. Konsum-)Zwänge sind eher hinderlich und eine Befreiung von diesen für ein glückliches Leben eher förderlich? Aus Gründen der Identitätsbildung ist ein von Nachhaltigkeit geprägter Lebensstil keineswegs für Schüler*innen aller Milieus naheliegend (Gärtner, 2020), dennoch kann ein passgenaues Begegnungslernen gewinnbringend sein. Die authentische Begegnung mit von Unrecht Betroffenen (etwa des bereits vorangeschrittenen Klimawandels) verbindet das abstrakte Thema mit konkreten Auswirkungen ausgewählter Einzelschicksale. Der einhergehende Perspektivwechsel befördert Empathie für das Leid anderer und die Sensibilisierung für dahinter liegende Ungerechtigkeit - hieraus kann auch der Wunsch nach Abhilfe und das Gefühl solidarischer Verantwortung erwachsen (Gaus, 2014, S. 38). Das (auch religionspädagogisch reflektierte) Feld des Globalen Lernens (z. B. Benk, 2019) liefert hier unersetzliche Impulse.

Stärkste Effekte auf die Entwicklung von Einstellungen dürften positive Gruppenerfahrungen in der Umsetzung von Nachhaltigkeitsengagement haben, was sowohl in kleinen Projekten im Kontext der Lerngruppe als auch als Whole School Approach auf Ebene der Schulentwicklung - gerade auch im Zusammenhang mit Schulpastoral (Birkel, 2019) - zu denken ist. Dabei wird nicht nur die Realisierbarkeit eines nachhaltigen Lebensstils im Sinne von Selbstwirksamkeit, sondern auch das mit dem solidarischen und sinn-schaffenden Tun häufig verbundene positive Gefühl erfahrbar. Der Leitgedanken „Schöpfungslust statt Ökofrust“ (Birkel, 2002, S. 267-269) erinnert an die häufig didaktisch außer Acht gelassene affektive Dimension von Einstellungen. Über die Reflexion eigenen Verhaltens erworbene bzw. veränderte Einstellungen zeigen sich zudem als besonders stabil. Auch ist das Erleben sog. IstNormen, also praktizierter Normen für das eigene Umweltverhalten überaus wichtig (Hamann, Baumann \& Löschinger, 2016, S. 46f.). Positive Erfahrungen alternativen Lebensstils sind eine vielversprechende Ausgangslage für die aus der Tugendethik bekannten Fragestellungen, die für Schüler*innen ad 
personam aufzuwerfen sind: „Wer bin ich, wie will ich sein, was benötige ich für gelingendes Leben?“ Für intellektuell fordernde Gruppen sind gesellschaftliche Konzepte wie Buen vivir (Welthaus Bielefeld, 2012; Rieckmann, 2017) ebenso diskurseröffnend wie gesellschaftsanalytische Ansätze wie die Theorie der Beschleunigungsgesellschaft und die Suche nach Resonanz (Hartmut Rosa) oder dem Suffizienzgedanken im Rahmen einer Postwachstumsökonomie (Niko Paech).

(ad 3) Dem dritten Einstellungskomplex ist zunächst erneut mit fragender Haltung und entsprechender Methodik zu begegnen (a). Worauf gründet diese Einschätzung, mit welchen anderen Bedenken ist diese Haltung assoziiert, welches Bild des Religionsunterrichts und welches Verständnis des christlichen Glaubens verbinden sich damit? Liegt die abweisende Einstellung ggf. in der Befürchtung, einer moralisierenden und von Appellen geprägten Kommunikationssituation ausgesetzt zu sein? In den sachlich vermittelnden Teil (b) fällt der Aufweis, dass die Bewerkstelligung von Mitigation eine primär kulturelltransformative Aufgabe ist, die unmöglich allein durch Wissenschaft und Technik zu leisten ist, sondern vielmehr normativer Reflexion und sinnstiftender Kommunikation bedarf. Zu klären ist zudem, dass christlicher Glaube stets eine gesellschaftlich-transformative und damit auch politische Dimension hat: Schöpfungstheologie (als prophetische Vision von Gerechtigkeit; Benk, 2016), Jesu Botschaft vom Reich Gottes als eine bessere Welt - diese zentralen biblisch-theologischen Topoi gilt es im Kontext der Klimaproblematik auszudeuten. Angesichts des christlichen Glaubens geht es also um eine konkrete Um- und Mitweltgestaltung. Auch die Beschäftigung mit kirchlichen Lehrschreiben der Evangelischen und Katholischen Kirche in Deutschland zu Ökologie und Klimaschutz, nicht zuletzt Papst Franziskus Mitweltenzyklika „Laudato si““ (2015) belegen eindrücklich das Interesse und die profilierte systemkritische Parteinahme aus dem christlichen Glauben heraus. Aufschlussreich dürfte für Schüler*innen auch die darin vorliegende (und den rein natur- oder sozialwissenschaftlichen Herangehensweisen i. d. R. ermangelnden) Problematisierung der anthropozentrischen Sicht auf Natur sein, nach der diese als verfügbare Ressource betrachtet wird, und es gilt zu diskutieren, ob „eine Sicht der Natur als Schöpfung helfen könnte, das nichtmenschliche Sein als Selbstzweck zu betrachten." (Bederna 2020a, Abs. 2). In einem dritten Schritt (c) kann eine neue Perspektiveinnahme erfolgen, im Rahmen dieser die Schüler*innen Theologizität als positionell-partizipatorische Hermeneutik erfahren (Englert, 2017, S. 29), in deren Horizont die Lernenden ihr Welt- und Selbstverständnis auch theologisch deuten können (Dressler, 2017).

Neben Anknüpfungspunkten in der theologischen Anthropologie (z. B. Verantwortung vor Gott und Bezogenheit zur Erde), lässt sich in Bezug auf die Verantwortungsethik Dietrich Bonhoeffers beispielsweise die in der paulinischen Denkweise verankerten Phase der Transformation oder des Liminalen (Strecker, 1999) begreifen, um eine theologische (Welt-)Deutungsmöglichkeit nachvollziehbar zu machen. In Hinblick auf die Ritualtheorie u. a. Victor Turners kann Strecker das Leben der Christusglaubenden als eine Übergangsphase (liminale Phase) thematisieren, die nicht nur stabilisierend, sondern auch aktualisierend wirkt. Das Leben in Christus, das der/dem Glaubenden in der liminalen Phase zuteil wird, verweist „auf die im Christusereignis eröffnete und dann in der Initiation an den jeweiligen Subjekten reaktualisierte grundsätzliche Veränderung der Wirklichkeit sowohl in kognitiver, emotionaler wie in pragmatischer Hinsicht und steht insofern gewissermaßen für das ,gelebte Weltbild' der Christusgläubigen" (Strecker, 1999, S. 211). Mit Hilfe dieser re-aktualisierten Welt-Sicht ist demnach ein Verständnis für das Ethos im Sinne der Bonhoeffer'schen Theologie und das Leben in der Transformation möglich.

\section{Fazit}

Die sorgfältige Wahrnehmung der Schüler*inneneinstellungen wie in diesem Beitrag vorgestellt, bietet Lehrer*innen die Möglichkeit, die Thematisierung der Klimakrise zielorientiert vorzubereiten und auf mögliche Widerstände differenziert einzugehen. Hat der Religionsunterricht ein „Image-Problem“ 
(Wissner \& Schweitzer, 2019, S. 54), da den Schüler*innen zufolge unpassende Meinungen im Religionsunterricht unerwünscht seien, so bietet die Fokussierung auf die Schüler*inneneinstellungen gerade die Chance zur ersichtlichen Absage an die vermeintliche cancel culture des Religionsunterrichts.

In der ernsthaften und fachspezifischen Auseinandersetzung mit Bedenken und Einwänden lässt sich die Orientierung religiöser Bildung am Ziel der Selbstbestimmung als Selbst-, Sach- und Sozialkompetenz ausweisen und so auch zur Steigerung der Akzeptanz des Religionsunterrichts beitragen (Bucher, 2001, S. 95f.; Wissner \& Schweitzer, 2019, S. 57).

Der Religionsunterricht hat damit die Möglichkeit, „,Begründungen und Motivationen für ethisches Urteilen und moralisches Handeln zu verdeutlichen sowie Empathie und ethische Sensibilität zu stärken und die dazugehörigen Glaubensperspektiven [...] nachvollziehbar zu machen“ (Pirner, 2019, S. 182). Er bietet damit aber zugleich herausfordernde Anregungen zum Überdenken eigener Einstellungen als Beitrag zur Identitätsbildung für alle Schüler*innen. Unter diesen Vorzeichen lassen sich vielleicht auch Schüler*innen für einen neuen Blick gewinnen, in dem das Klima nicht nervt, sondern Neugier an den großen Zusammenhängen und der eigenen Person wecken kann.

\section{Literaturverzeichnis}

Bederna, Katrin (2020a). Art. Bildung für nachhaltige Entwicklung. Das wissenschaftlich-religionspädagogische Lexikon im Internet. https://doi.org/10.23768/wirelex.Bildung fr nachhaltige Entwicklung.$\underline{200572}$

Bederna, Katrin (2020b). Every Day for Future. Theologie und religiöse Bildung für nachhaltige Entwicklung (2. Auflage). Ostfildern: Matthias Grünewald.

Bederna, Katrin \& Krahn, Annika (2021). Art. Klimawandel. Das wissenschaftlich-religionspädagogische Lexikon im Internet. https://doi.org/10.23768/wirelex.Klimawandel.200896

Benk, Andreas (2016). Schöpfung - eine Vision von Gerechtigkeit. Was niemals war, doch möglich ist. Ostfildern: Matthias Grünewald.

Benk, Andreas (2019). Globales Lernen als Kernaufgabe religiöser Bildung. In Andreas Benk (Hg.), Globales Lernen. Bildung unter dem Leitbild weltweiter Gerechtigkeit (S. 213-224). Ostfildern: Matthias Grünewald.

Birkel, Simone (2002). Zukunft wagen - ökologisch handeln. Grundlagen und Leitbilder kirchlich-ökologischer Bildung im Kontext nachhaltiger Entwicklung. Münster: LIT

Birkel, Simone (2019). Gutes Leben ermöglichen! Nachhaltigkeit und Schöpfungsverantwortung im Kontext von Schulpastoral. In Sabine Bieberstein, Peter Nothaft \& Barbara Staudigl (Hg.), Hoffnungsraum Schule. Perspektiven für eine profilierte Entwicklung katholischer Schulen (S. 55-67). Ostfildern: Matthias Grünewald.

Brunnengräber, Achim (2013). Klimaskeptiker in Deutschland und ihr Kampf gegen die Energiewende. Berlin: FFU-report.

Bucher, Anton (2001). Religionsunterricht zwischen Lernfach und Lebenshilfe. Eine empirische Untersuchung zum katholischen Religionsunterricht in der Bundesrepublik Deutschland (3. Auflage). Stuttgart: Kohlhammer.

Bucher, Anton A. (2019). Ist Interesse geweckt, läuft das meiste von selbst - Interesse und Desinteresse im und am Religionsunterricht. In Stefan Altmeyer, Bernhard Grümme, Helga Kohler-Spiegel, Elisabeth Naurath, Bernd Schröder \& Friedrich Schweitzer (Hg.), Reli - keine Lust und keine Ahnung? Jahrbuch der Religionspädagogik 35 (S. 34-45). Göttingen: Vandenhoeck \& Ruprecht.

Bundesministerium für Umwelt, Naturschutz und nukleare Sicherheit [BMU] (Hg.) (2018). Zukunft? Jugend fragen! Nachhaltigkeit, Politik, Engagement - eine Studie zu Einstellungen und Alltag junger Menschen. Berlin: Bundesministerium für Umwelt, Naturschutz und nukleare Sicherheit. 
Bundesministerium für Umwelt, Naturschutz und nukleare Sicherheit [BMU] (Hg.) (2020). Zukunft? Jugend fragen! Umwelt, Klima, Politik, Engagement - Was junge Menschen bewegt. Berlin: Bundesministerium für Umwelt, Naturschutz und nukleare Sicherheit.

Comenius-Institut (Hg.) (2019). Evangelischer Religionsunterricht. Empirische Befunde und Perspektiven aus Baden-Württemberg, Niedersachsen und Sachsen. Münster: Waxmann.

The Climate Outreach and Information Network [COIN] (Eds.) (2014). How do young people engage with climate change? Towards more effective communication with 18-25 year olds. London: Creative Commons.

Deutsche Shell Holding GmbH (Hg.) (2019). Jungend 2019 - 18. Shell Jugendstudie. Eine Generation meldet sich zu Wort. Weinheim: Beltz.

Dressler, Bernhard (2017). Warum und auf welche Weise Religionspädagogik als Theologie zu betreiben ist. Überlegungen zur Theologizität der Religionspädagogik. In Thomas Schlag \& Jasmine Suhner (Hg.), Theologie als Herausforderung religiöser Bildung. Bildungstheoretische Orientierungen zur Theologizität der Religionspädagogik (S. 85-100). Stuttgart: Kohlhammer.

Englert, Rudolf (2017). Das Theologische der Religionspädagogik. Grundfragen und Herausforderungen. In Thomas Schlag \& Jasmine Suhner (Hg.), Theologie als Herausforderung religiöser Bildung. Bildungstheoretische Orientierungen zur Theologizität der Religionspädagogik (S. 21-32). Stuttgart: Kohlhammer.

Enzian, Annett (2015). Denn sie tun nicht, was sie wissen. Eine Studie zu ökologischem Bewusstsein und Handeln. München: oekom-Verlag.

Fuchs, Monika E. (2019). Wer hat kein Interesse am Religionsunterricht? In Stefan Altmeyer, Bernhard Grümme, Helga Kohler-Spiegel, Elisabeth Naurath, Bernd Schröder \& Friedrich Schweitzer (Hg.), Reli - keine Lust und keine Ahnung? Jahrbuch der Religionspädagogik 35 (S. 92-111). Göttingen: Vandenhoeck \& Ruprecht.

Gärtner, Claudia (2020). Klima, Corona und das Christentum. Religiöse Bildung für nachhaltige Entwicklung in einer verwundeten Welt. Bielefeld: transcript Verlag.

Gaus, Ralph (2014). Globales Lernen im Religionsunterricht als Beitrag zur Persönlichkeitsentwicklung. In Institut für Religionspädagogik der Erzdiözese Freiburg (Hg.), Globales Lernen (IRP Impulse 1/2014) (S. 36-39). Freiburg: IfR.

Gerhold, Lars (2020). COVID-19: Risk perception and Coping Strategies. Results from a survey in Germany. Berlin: FU Berlin. https://doi.org/10.31234/osf.io/xmpk4

Hamann, Karen; Baumann, Anna \& Löschinger, Daniel (2016). Psychologie im Umweltschutz. Handbuch zur Förderung nachhaltigen Handelns. München: oekom-Verlag.

Havers, Norbert (1972). Der Religionsunterricht - Analyse eines unbeliebten Fachs. Eine empirische Untersuchung. München: Kösel.

Kommission Weltkirche der Deutschen Bischofskonferenz (Hg.) (2021). Wie sozial-ökologische Transformation gelingen kann. Eine interdisziplinäre Studie im Rahmen des Dialogprojektes zum weltkirchlichen Beitrag der katholischen Kirche für eine sozial-ökologische Transformation im Lichte von Laudato si' (Studien der Sachverständigengruppe „Weltwirtschaft und Sozialethik“ 22). Bonn: Eigenverlag

Masson-Delmotte, Valérie; Zhai, Panmao; Pirani, Anna; Connors, Sarah L.; Péan, Clotilde; Berger, Sophie; Caud, Nada; Chen, Yang; Goldfarb, Leah; Gomis, Melissa I.; Huang, Mengtian; Leitzell, Katherine; Lonnoy, Elisabeth; Matthews, Robin; Maycock, Thomas K.; Waterfield, Tim; Yelekçi, Ozge; Yu, Rong \& Zhou, Baiquan (2021). Climate Change 2021: The Physical Science Basis. Contribution of Working Group I to the Sixth Assessment Report of the Intergovernmental Panel on Climate Change. Cambridge: Cambridge University Press.

Pirner, Manfred L. (2019). Welche Kompetenzen brauchen Religionslehrkräfte im Umgang mit religionsdistanten Schülerinnen und Schülern? In Stefan Altmeyer, Bernhard Grümme, Helga KohlerSpiegel, Elisabeth Naurath, Bernd Schröder \& Friedrich Schweitzer (Hg.), Reli - keine Lust und keine Ahnung? Jahrbuch der Religionspädagogik 35 (S. 173-183). Göttingen: Vandenhoeck \& Ruprecht. 
Prawdzik, Robert (1973). Der Religionsunterricht im Urteil der Hauptschüler. Eine empirische Untersuchung auf der 9. Klasse Hauptschule in München. Zürich: Benziger.

Reif, Alexander \& Heitfeld, Marie (2015). Wandel mit Hand und Fuß. Mit dem Germanwatch Hand Print den Wandel politisch wirksam gestalten. Bonn: Germanwatch. http://germanwatch.org/de/12040

Rieckmann, Marco (2017). Bildung für nachhaltige Entwicklung in der Großen Transformation - Neue Perspektiven aus den Buen-Vivir- und Postwachstumsdiskursen. In Oliver Emde, Uwe Jakubczyk, Bernd Kappes \& Bernd Overwien (Hg.), Mit Bildung die Welt verändern? Globales Lernen für eine nachhaltige Entwicklung (S. 147-159). Opladen: Barbara Budrich.

Ritzer, Georg (2010). Interesse - Wissen - Toleranz - Sinn. Ausgewählte Kompetenzbereiche und deren Vermittlung im Religionsunterricht. Eine Längsschnittstudie. Berlin: LIT.

Rosenberger, Michael (2014). Die Ratio der „Klima-Religion“. Eine theologisch-ethische Auseinandersetzung mit klimaskeptischen Argumenten. GAIA - Ecological Perspectives for Science and Society, 23(2), 93-99. https://doi.org/10.14512/gaia.23.2.6

Roth, Heinrich (1971). Pädagogische Anthropologie. Band II: Einwicklung und Erziehung. Grundlagen einer Entwicklungspädagogik. Hannover: Schroedel.

Rumberger, Achim (2021). Steter Tropfen höhlt den Stein. Wie Jahrzehnte der Wissenschaftsleugnung unsere Gesellschaft gefährden. Norderstedt: Books on Demand.

Schimmel, Alexander (2011). Einstellungen gegenüber Glauben als Thema des Religionsunterrichts. Didaktische Überlegungen und Anregungen für die gymnasiale Oberstufe. Ostfildern: Schwabenverlag

Schmid, Philipp (2020). Effective Strategies to Counter Science Denialism in Public. Erfurt: Universität Erfurt. https://www.db-thueringen.de/receive/dbt mods 00045806

Sekretariat der Ständigen Konferenz der Kultusminister der Länder in der Bundesrepublik Deutschland [KMK] (Hg.) (2002). Zur Situation des Evangelischen Religionsunterrichts in der Bundesrepublik Deutschland. Berlin: Wissenschaftsforum.

Sinus Studie (2019). SINUS-Studie zu Fridays for Future und Klimaschutz. Heidelberg: SINUS.

Strecker, Christian (1999). Die liminale Theologie des Paulus. Zugänge zur paulinischen Theologie aus kulturanthropologischer Perspektive. Göttingen: Vandenhoeck \& Ruprecht.

Vogt, Markus (2021). Christliche Umweltethik. Grundlagen und zentrale Herausforderungen. Freiburg: Herder

Weis, Mirjam; Reiss, Kristina; Mang, Julia; Schiepe-Tiska, Anja; Diedrich, Jennifer; Roczen, Nina \& Jude, Nina (2020). Global Competence in PISA 2018. Einstellungen von Fünfzehnjährigen in Deutschland zu globalen und interkulturellen Themen. Münster: Waxmann.

Welthaus Bielefeld (Hg.) (2012). »Buen Vivir - Was heißt Gutes Leben? « Bildungsmaterial für die Fächer Praktische Philosophie und Religion (Kl. 9+10). Bielefeld: Welthaus Bielefeld.

Werth, Lioba; Denzler, Markus \& Mayer, Jennifer (2020). Sozialpsychologie - Das Individuum im sozialen Kontext. Wahrnehmen - Denken - Fühlen (2. Auflage). Berlin: Springer.

Wilts, Henning \& Fecke, Marina (Hg.) (2020). ReUse und Secondhand in Deutschland. Einstellungen zum Thema Abfallvermeidung und Nachhaltigkeit. Wuppertal: Wuppertal Institut.

Wissenschaft im Dialog gGmbH (Hg.) (2016). Wissenschaftsbarometer 2016. Berlin: WiD.

Wissner, Golde \& Schweitzer, Friedrich (2019). Wen der Religionsunterricht nicht erreicht und wie er sich darum ändern müsste - Schülerwahrnehmungen zum Religions- und Ethikunterricht im Vergleich. In Stefan Altmeyer, Bernhard Grümme, Helga Kohler-Spiegel, Elisabeth Naurath, Bernd Schröder \& Friedrich Schweitzer (Hg.), Reli - keine Lust und keine Ahnung? Jahrbuch der Religionspädagogik 35 (S. 46-61) Göttingen: Vandenhoeck \& Ruprecht. 